\title{
Plano "Viver sem Limite" e a Rede de Cuidados da Pessoa com Deficiência: representações em um cenário da Fronteira Oeste do Rio Grande do Sul
}

\author{
Christian Caldeira Santos \\ Universidade Federal do Pampa (UNIPAMPA) - Uruguaiana - RS - Brasil \\ ORCID: 0000-0001-6394-5288 \\ Jacinta Sidegum Renner \\ Universidade Feevale - Novo Hamburgo - RS - Brasil \\ ORCID: 0000-0002-9904-4710 \\ Gustavo Roese Safelice \\ Universidade Feevale - Novo Hamburgo - RS - Brasil \\ ORCID: 0000-0003-0159-358 \\ Susane Graup \\ Universidade Federal do Pampa (UNIPAMPA) - Uruguaiana - RS - Brasil \\ ORCID: 0000-0002-3389-8975 \\ Rodrigo de Souza Balk \\ Universidade Federal do Pampa (UNIPAMPA) - Uruguaiana - RS - Brasil \\ ORCID: 0000-0001-5254-6732
}

\begin{abstract}
Resumo
O Plano Nacional dos Direitos da Pessoa com Deficiência - "Viver sem Limite", eixo saúde, por meio da Rede de Cuidados da Pessoa com Deficiência (RCPD) trouxe perspectivas de melhorias à saúde dos brasileiros com deficiência. Assim, objetiva-se analisar e verificar a interferência do Plano "Viver sem Limite" junto da RCPD sobre o processo de inclusão social de crianças com Paralisia Cerebral (PC) em Uruguaiana, Fronteira Oeste do Rio Grande do Sul. A pesquisa se caracteriza como aplicada, descritiva e de estudo de caso, sob o paradigma qualitativo associada à pesquisa documental de fonte primária. Intencionalmente, selecionou-se 16 pais/responsáveis de crianças com PC de dois serviços de reabilitação física infantil no município. Eles responderam dois questionários (um estruturado e ou semiestruturado), sendo as entrevistas gravadas, transcritas e analisadas por meio da análise de conteúdo sob o modelo da análise temática. Os resultados apontaram que todos desconhecem a existência do Plano e da RCPD tanto a nível local, estadual e nacional. Há falha na assistência às crianças em todos os níveis de atenção da
\end{abstract}


saúde e intensa participação de atores e/ou figuras ocultas na Rede local. Positivamente ocorreu a concessão de órteses e meios de locomoção às crianças com PC. Assim, o Plano "Viver sem Limite" por meio da RCPD municipal não contemplam a integralidade no tratamento de reabilitação das crianças com PC e precisa ser aperfeiçoado para oportunizar melhores cuidados à saúde a essas crianças.

Palavras-chave: Políticas públicas. Inclusão Social. Saúde. Deficiência. Paralisia Cerebral.

\section{"Living Without Limit" Plan and the Care Network for People with Disabilities: representations in a scenario of the Western Frontier of Rio Grande do Sul}

\section{Abstract}

The National Plan for the Rights of Persons with Disabilities - "Living without Limit", the health axis, through the Care Network for Persons with Disabilities (CNPD) brought prospects for improving the health of brazilians with disabilities. Thus, the objective is to analyze and verify the interference of the "Living without Limit" Plan with the CNPD on the process of social inclusion of children with Cerebral Palsy (CP) in Uruguaiana, Western Frontier of Rio Grande do Sul. The research is characterized as applied, descriptive and case study, under the qualitative paradigm associated with documentary research of primary source. Intentionally, 16 parents / guardians of children with CP were selected from two child physical rehabilitation services in the municipality. They answered two questionnaires (one structured and or semi-structured), the interviews being recorded, transcribed and analyzed through content analysis under the thematic analysis model. The results showed that everyone is unaware of the existence of the Plan and the CNPD both at the local, state and national levels. There is a failure to assist children at all levels of health care and intense participation by actors and / or hidden figures in the local network. Positively, orthosis and means of locomotion were granted to children with CP. Thus, the "Live without Limit" Plan, through the municipal CNPD, does not contemplate integrality in the rehabilitation treatment of children with $\mathrm{CP}$ and needs to be improved to provide better health care to these children.

Keywords: Public policies. Social inclusion. Health. Disability. Cerebral Palsy.

\section{Plan "Vivir sin límite" y la Red de atención para personas con discapacidad: representaciones en un escenario de la frontera occidental de Rio Grande do Sul \\ Resumen}

El Plan Nacional para los Derechos de las Personas con Discapacidad: "Vivir sin Límites", el eje de salud, a través de la Red de Atención a las Personas con Discapacidad (RAPD) trajo perspectivas para mejorar la salud de los brasileños con discapacidad. Por lo tanto, el objetivo es analizar y verificar la interferencia del Plan "Vivir sin Límite" con el RAPD en el proceso de inclusión social de niños con parálisis cerebral $(P C)$ en Uruguaiana, frontera occidental de Rio Grande do Sul. La investigación se caracteriza como aplicada, estudio descriptivo y de caso, bajo el paradigma cualitativo asociado a la investigación documental de fuente primaria. Intencionalmente, 16 padres / tutores de niños con PC fueron seleccionados de dos servicios de rehabilitación física infantil en el municipio. Respondieron dos cuestionarios (uno estructurado y / o semiestructurado), las entrevistas fueron grabadas, transcritas y analizadas a través del análisis de contenido bajo el modelo de análisis temático. Los resultados mostraron que todos desconocen la existencia del Plan y el RAPD a nivel local, estatal y nacional. Hay una falla en ayudar a los niños en todos los niveles de atención médica y una intensa participación de actores y/o figuras ocultas en la red local. Positivamente, se otorgaron órtesis y medios de locomoción a niños con PC. Por lo tanto, el Plan "Vivir sin Límite", a través del RAPD municipal, no contempla la integralidad en el tratamiento de rehabilitación de niños con PC y necessita ser mejorado para brindar una mejor atención de salud a estos niños. 
Palabras clave: Políticas públicas. Inclusión social. Salud. discapacidad. Parálisis cerebral.

\section{Introdução}

O Plano Nacional dos Direitos da Pessoa com Deficiência - "Viver sem Limite" tensiona políticas, programas e ações perfazendo o exercício pleno e equitativo dos direitos das pessoas com deficiência em todo território brasileiro. Ele alicerçou suas nuances nas prerrogativas da Convenção Internacional sobre os Direitos das Pessoas com Deficiência e se apresenta em quatro eixos de atuação: educação, saúde, inclusão social e acessibilidade (BRASIL, 2011a).

No eixo saúde, o Ministério da Saúde (MS) criou a Rede de Cuidados da Pessoa com Deficiência (RCPD) no âmbito do Sistema Único de Saúde (SUS) pela Portaria $n^{\circ} 793$ em 2012. Ela buscou apoio entre os três pilares da Rede de Atenção à Saúde: a Atenção Básica (AB), a Atenção Especializada em Reabilitação (AER) e a Atenção Hospitalar e de Urgência e Emergência (AHUE) (BRASIL, 2012a).

Intrínseco a essa Rede, a ideia da integralidade do cuidado ao público com deficiência baliza-se em "ações e serviços articulados entre si que favoreçam as parcerias entre os diversos serviços e atores da rede, o financiamento adequado, além do comprometimento de trabalhadores e gestores de saúde para lidar com as deficiências" (DUBOW; GARCIA; KRUG, 2018, p. 465).

Especificamente no Rio Grande do Sul (RS), o município de Uruguaiana aderiu ao Plano "Viver sem Limite" em agosto de 2013 (BRASIL, 2013a). O município encontra-se em uma região pampeana, quase extremo da Fronteira Oeste, caracterizada por indicadores sociais relacionados à saúde, renda e educação que transitam de valores baixos a medianos.

Segundo o Plano Municipal de Saúde de Uruguaiana (2018 - 2021) os serviços da rede saúde municipal são ofertados na Atenção Primária - $A B$ (Estratégia Saúde da Família [ESF], Núcleo de Apoio a Saúde da Família [NASF], Consultório de Rua, Oficinas Terapêuticas, Serviço de Ginecologia - Obstetrícia, Programa Saúde e Prevenção na Escola, Projeto Operativo Local, Bolsa Família, Saúde do Idoso, Primeira Infância Melhor [PIM], Atendimento a Estrangeiros e Programa de Melhoria do Acesso e da Qualidade da Atenção Básica), Atenção Secundária - Média Complexidade (Brasil Sorridente e Centro de Especialidades Odontológicas [CEO], Policlínica Municipal, Policlínica Infantil, Serviços de Fisioterapia, Rede de Atenção Psicossocial, Laboratório de Análises Clínicas, Clínica Renal, Banco de Sangue, Ambulatório de Especialidades Médicas do Hospital Santa Casa de Caridade de Uruguaiana [HSCCU] e Tratamento Fora do Domicílio [TFD]) e Atenção Terciária - Alta complexidade (Serviço de Oncologia, Cirurgia Cardiovascular, Neurologia e Neurocirurgia no HSCCU, Unidade de Pronto Atendimento - [UPA] 24 horas e Serviço de Atendimento Móvel de Urgência [SAMU]). Concomitantemente, estão presentes os serviços de Vigilância Epidemiológica, Sanitária, Ambiental e em Saúde do Trabalhador (URUGUAIANA, 2017).

Nesse universo biossocioeconômico encontram-se crianças com Paralisia Cerebral (PC). Segundo Rosenbaum et al. (2006), a PC está atrelada a um coletivo de alterações do desenvolvimento da ação motora e da postura que debilita a atividade funcional humana. Essas alterações podem ou não ser acompanhadas de distúrbios de sensação, percepção, cognição, comunicação, comportamento, epilepsia e lesões osteomusculares secundárias; e sua origem está condicionada a uma lesão encefálica não progressiva no período do desenvolvimento encefálico 
fetal ou infantil.

Diante desse espectro disfuncional, Rotta (2002) apontou que as crianças com PC devam ser assistidas por uma equipe multidisciplinar em saúde. Para o MS, elas carecem de uma rede de cuidados imbricada na assistência global à saúde com vistas à estruturação de um projeto terapêutico de qualidade, que une equipes de saúde e família, cujo somatório de ações promova ampliação da autonomia e inclusão social dessa parcela populacional (BRASIL, 2013b).

Assim, questiona-se: Qual(is) repercussão(ões) que a RCPD proporcionou às crianças com PC de Uruguaiana? Para tanto, objetiva analisar e verificar a interferência do Plano "Viver sem Limite" por meio da RCPD sobre o processo de inclusão social" de crianças com PC residentes em Uruguaiana.

\section{Materiais e Método}

Esta pesquisa se caracteriza como aplicada, descritiva e de estudo de caso sob o paradigma qualitativo, associada à pesquisa documental de fonte primária (PRODANOV; FREITAS, 2013), realizada em um universo de 26 pais/responsáveis de crianças com PC que frequentavam dois locais de reabilitação física infantil em Uruguaiana. Ela apresentou amostragem não probabilística, por conveniência, visto os dois locais serem referências no município para o tratamento fisioterapêutico de crianças com PC. Foram incluídos na pesquisa os pais/responsáveis de crianças com diagnóstico clínico de PC com idade cronológica entre 04 e 12 anos que usufruíam dos serviços de fisioterapia desses dois locais de reabilitação no município e residentes nele por no mínimo um ano. Foram excluídos os pais/responsáveis de crianças com PC, as quais sofreram intervenção médica invasiva nos últimos seis meses antes da entrevista realizada, e aqueles que possuíram dificuldades em responder os questionários de pesquisa. Ao final, 16 pais/responsáveis de crianças com diagnóstico clínico de PC foram elegíveis para participar e assim constituíram a amostra da pesquisa.

Quanto aos procedimentos éticos, os participantes foram contatados pessoalmente nos locais de tratamento e foram convidados a participarem do estudo. Os que aceitaram foram solicitados a assinar o Termo de Consentimento Livre e Esclarecido. Posteriormente, um questionário estruturado relacionado ao perfil biodemográfico e outro semiestruturado foi aplicado aos pais/responsáveis. As entrevistas tiveram as falas dos pais/responsáveis gravadas, transcritas e analisadas pela técnica de Análise de Conteúdo sob o modelo da Análise Temática. Essa técnica é dividida em três partes: pré-análise, exploração do material e tratamento dos resultados obtidos com sua interpretação em categorias (MINAYO, 2014). Seguidamente, as análises foram tencionadas com as informações contidas no Plano Municipal de Saúde (2018-2021) de Uruguaiana (URUGUAIANA, 2017).

Assim, os dados foram descritos quanto ao perfil biodemográfico dos pais/responsáveis das crianças com PC e a Análise Temática dos dados gerou três

${ }^{1} O$ termo inclusão social foi usado sob a premissa de Sassaki (2009), que são as ações promovidas por meio de uma engrenagem sistêmica social que disponibiliza oportunidades destinadas ao conjunto heterogêneo humano, sendo que o criador, preceptor e executor dessas oportunidades possuem esse próprio conjunto. 
temáticas: O balanço da rede...da Rede de Cuidados da Pessoa com Deficiência de Uruguaiana; Atores e/ou figuras ocultas da Rede de Cuidados da Pessoa com Deficiência de Uruguaiana e a Interferência da Rede de Cuidados da Pessoa com Deficiência na inclusão social de crianças com Paralisia Cerebral. Sinaliza-se que para preservar a identidade dos participantes foi utilizado o termo "responsável por" para a identificação dos pais/responsáveis correspondentes, acrescido de um nome fictício da criança (deuses da mitologia grega).

A coleta de dados ocorreu no segundo semestre de 2018 e fevereiro de 2019, nos dias do tratamento fisioterapêutico das crianças, nas dependências dos dois locais de reabilitação física da cidade, tendo a pesquisa aprovada pelo Comitê de Ética em Pesquisa da Universidade Feevale, CAAE 98591218.3.0000.5348, em 17 de outubro de 2018, parecer número 2965.260, como determina a Resolução 466/12 do Conselho Nacional de Saúde que trata dos aspectos éticos em pesquisa envolvendo seres humanos no país (BRASIL, 2012b).

\section{Resultados e Discussão}

Diante dos critérios de inclusão/exclusão aplicados ao universo de 26 pais/responsáveis de crianças com PC foram elegíveis 16 participantes. O grupo foi composto por 13 mães, dois pais e uma avó paterna, tendo resultado similar quanto à distribuição de quem era o cuidador principal desse grupo de crianças. Dois entrevistados (uma mãe e um pai) não se identificaram como o cuidador principal. As principais características dos cuidadores principais estão demonstradas no Quadro 1.

Quadro 1 - Perfil biodemográfico dos cuidadores principais de crianças com paralisia cerebral de dois centros de reabilitação física infantil de Uruguaiana

\begin{tabular}{|c|c|c|c|c|c|}
\hline $\begin{array}{l}\text { Faixa Etária } \\
\text { (anos) }\end{array}$ & $\begin{array}{c}\text { Estado } \\
\text { Civil }\end{array}$ & $\begin{array}{l}\text { Nível } \\
\text { Escolar }\end{array}$ & Ocupação & $\begin{array}{c}\text { Classe Social / } \\
\text { ABEP (2019) }\end{array}$ & $\begin{array}{l}\text { Plano de } \\
\text { Saúde }\end{array}$ \\
\hline $20-30=8$ & Solteiro $=6$ & $E F=8$ & Do lar $=10$ & $A=3$ & $\operatorname{Sim}=5$ \\
\hline $31-40=6$ & Casado $=5$ & $E M=6$ & Contadora $=1$ & $B 2=4$ & Não = 11 \\
\hline$>50=2$ & Outros $=5$ & $E S=2$ & $\begin{array}{c}\text { Autônomo }=2 \\
\text { Atendente }=1 \\
\text { Trabalhador } \\
\text { rural }=1 \\
\text { Estudante }=1\end{array}$ & $\begin{array}{l}C_{1}=3 \\
C_{2}=3 \\
D=3\end{array}$ & \\
\hline
\end{tabular}

Legenda: EF - Ensino Fundamental; EM - Ensino Médio; ES - Ensino Superior; ABEP - Associação Brasileira de Empresas de Pesquisa - Critérios Brasil 2019

Fonte: conforme dados do autor (2019)

A maioria das famílias (69\%) são usuárias do SUS para o tratamento de seu dependente com PC. As crianças com PC estão inseridas em grupos familiares com menor nível socioeconômico (56\%), com baixa e média escolaridade (87,5\%) e com a função ocupacional do cuidador principal atribuída aos cuidados domésticos (62,5\%). Estes resultados se assemelham ao do estudo de Coimbra e Müller (2017), onde prevaleceu cuidadores do sexo feminino e de classe socioeconômica baixa; ao 
de Dantas et al. (2017), onde apresentou maior número de cuidadores com atividades relacionadas ao lar e com famílias dependentes do SUS; e ao de Santos, Marques e Souza (2017), no qual houve maior frequência de famílias em que os cuidadores, no máximo, apresentavam o ensino fundamental completo.

\subsection{O balanço da rede... da Rede de Cuidados da Pessoa com Deficiência de Uruguaiana.}

Após análise do Plano Municipal de Saúde 2 (2018 - 2021) de Uruguaiana (URUGUAIANA, 2017), percebeu-se que a RCPD não está descrita e talvez não constituída oficialmente, embora, o município tenha aderido ao Plano "Viver sem Limite" em agosto de 2013 (BRASIL, 2013a) e atualmente, a Secretaria Estadual da Saúde do RS aponte o município como referência para a Triagem Auditiva Neonatal e, o seu Serviço de Fisioterapia esteja cadastrado no SUS, como atendimento em modalidade única para deficiência física (RIO GRANDE DO SUL, 2019).

Nesse entrave organizacional e estrutural, relata-se que no decorrer do texto, ao se mencionar a RCPD de Uruguaiana, entenda-se essa como uma Rede "extraoficial", construída a partir das narrativas dos 16 pais/responsáveis e somada aos dados municipais obtidos por meio da análise do seu Plano Municipal de Saúde (2018-2021).

De início, destacou-se que todos os pais/responsáveis desse grupo de 16 crianças com PC asseguraram desconhecer a existência do Plano "Viver sem Limite" e, concomitantemente, da RCPD no âmbito do SUS, seja, a nível municipal, estadual ou nacional. Fato contrário foi noticiado por Dubow, Garcia e Krug (2018), onde a existência do serviço de reabilitação de referência da $28^{\circ}$ Região de Saúde do RS propiciou sua lembrança pelos usuários que o classificou como uma potencialidade da RCPD. Assim, quando seus setores se apresentam visíveis e operantes, a RCPD se consolida como uma memória de referência e de existência para os usuários do SUS.

Menciona-se, que um dos 16 pais/responsáveis demonstrou surpresa de a RCPD estar integrada ao SUS, conforme pode ser vista na narrativa a seguir:

Você conhece o Plano Nacional da Pessoa com Deficiência - "Viver sem Limite" e a Rede de Cuidados da Pessoa com Deficiência? <Pesquisador>.

“[...] Não! Aonde? [...]”. <Responsável por Hércules>.

Você o usa no SUS! <Pesquisador>.

"[...] Como? Por que nunca ninguém chegou para mim e disse isso? [...]" $<$ Responsável por Hércules >.

Entende-se que uma comunicação pobre e não efetiva resulta em informações não favoráveis à população. Sob esse aspecto, Othero e Ayres (2012) relatam que a informação e a orientação compõem aspectos relacionados ao direito e a cidadania da pessoa com deficiência. Revela-se que Teixeira (2004), já havia

\footnotetext{
${ }^{2}$ Plano de Saúde é "um documento de intenções políticas, diagnósticos, estratégias, prioridades e metas [...] que consolida o planejamento de saúde para um determinado período em cada esfera de governo" (FIGUEIREDO et al., 2007, p. 43).
} 
descrito que a comunicação ligada ao ramo da saúde pode favorecer o uso dos serviços e recursos de saúde, assim como ampliar o acesso aos serviços dessa natureza pela população.

Nesse bojo, a ausência de informação dos pais/responsáveis se deve por falta de comunicação em saúde, ou seja, a rede municipal de saúde não fez uso de estratégias e ações para fomentar decisões individuais ou coletivas com vista à promoção da saúde local da pessoa com deficiência. Percebe-se um desalinhamento com a informação, onde a resultante é a desassistência ou a assistência parcial das crianças com PC.

Quanto aos motivos relatados para o desconhecimento da RCPD, eles transitam pela não informação oferecida por órgãos oficiais ou pelos locais de tratamento, por profissionais da área de reabilitação, por outras famílias ou de si próprios, conforme pode ser visualizado nas descrições a seguir:

“[...] Nunca ouvir falar! [...]” <Responsável por Apolo>.

"[...] É falta de informação mesmo, porque muito que a gente aprende é o que a gente corre atrás ou o que alguém vem e fala: olha existe tal coisa, existe tal programa [...]" <Responsável por Ajax>.

"[...] Olha ... quem sabe de ler mais os planos do governo, de buscar o conhecimento ou também é pouca divulgação, né! [...]” <Responsável por Édipo>.

A partir de 2019, as informações sobre o Plano "Viver sem Limite" e a RCPD se encontram no site do Ministério da Mulher, da Família e dos Direitos Humanos e do MS e no da Secretaria Estadual da Saúde do RS. Este último oferece informações sobre a estrutura e organização da RCPD estadual. Assim, infere-se que a desinformação, tanto do Plano como da RCPD, constrói e exacerba obstáculo para a não inclusão social desse grupo de crianças com PC no cenário municipal estudado.

Ao mesmo instante, os relatos dos 16 participantes evidenciam algumas articulações de ações e estratégias nos três pontos ( $A B, A E R$ e AHUE) de organização da RCPD local com vista à identificação do processo de inclusão social de seus familiares. Segundo Mendes (2014, p. 151), essas articulações podem demonstrar "a materialização de uma conquista histórica em termos de direitos sociais e, neles, de acesso qualificado das pessoas com deficiência à saúde".

$\mathrm{Na} A B$ da RCPD local, dez pais/responsáveis relataram utilizar Unidades Básicas de Saúde (UBS) para consultas médicas e todos para vacinações das suas crianças.

Quanto as ações dos agentes de saúde nos territórios das UBS's, houve relatos de que confirmaram e negaram suas ações relacionadas ao tema deficiência, conforme descrições a seguir:

“[...] Sempre perguntam! E sempre que eu necessitei de consulta, o agente que vai na minha casa dá um jeitinho daqui, dali e arruma a consulta. [...]" < Responsável por |ris>.

"[...] Sim, já foram uma vez na minha casa. Ficaram de ir visitar, mas depois nunca mais voltaram! [...]" <Responsável por Poseidon>.

"[...] Não! Nunca, nunca, nunca! [...]. De idoso eu já vi, mas assim de criança com necessidade, assim não! [...]" < Responsável por Hipnos>. 
$\mathrm{Na}$ premissa da $A B$ "ser a principal porta de entrada e centro de comunicação da Rede de Atenção à Saúde" (BRASIL, 2011b), esses relatos demonstram falhas nas ações em saúde nesse estágio da RCPD em Uruguaiana. Esse feito relaciona-se aos dados que apontam que até fevereiro de 2019 houve uma cobertura mediana (53\%) da ESF e de quase $69 \%$ da AB aos seus munícipes (BRASIL, 2019).

Um cenário semelhante foi descrito por Schultz e Alonso (2016) na cidade do Rio de Janeiro. Nele a fragilidade nas ações da $A B$ para com crianças com deficiências se tencionava à incoordenação do cuidado e o não desenvolvimento de uma atenção integral, fundamentada no apreço familiar e comunitário.

$\mathrm{Na}$ perspectiva uruguaianense, afirma-se que a presença de pessoas com deficiência nos territórios das UBS pode estar sub-referendada ou até mesmo não referendada, resultando em uma ideia da não necessidade de implantar ações e estratégias da atenção em saúde, que propicia tecer a rede de apoio com vistas ao processo de inclusão social desse público no município.

Relata-se também, que até 2017, Uruguaiana dispôs somente de um NASF (URUGUAIANA, 2017), o qual, de certo modo, contribuiu para o influxo de encaminhamento de pessoas com deficiência para o platô superior da RCPD que seria a AER, visto que Alves, Ribeiro e Sampaio (2016) relatam que com o advento dos NASF em Belo Horizonte - MG houve ganhos na RCPD, pois houve expansão nas estratégias entre as equipes da ESF, assim como de reabilitação.

Para o caso de Uruguaiana, essa ideia de multiplicação dos NASF's passa a ser uma opção para a tomada de ações e estratégias de saúde em prol da pessoa com deficiência. Assim, para o futuro, espera-se uma tendência contrária ao cenário da desassistência da pessoa com deficiência que vigorava no passado antes da criação do SUS, conforme foi relatada por Othero e Dalmaso (2009).

De acordo com a Portaria $n^{\circ} 793$ de 2012 do MS, nesse nível de assistência esperam-se ações estratégicas para a ampliação do acesso e da qualificação na atenção da pessoa com deficiência, pautada na identificação precoce das deficiências, vigilância infantil com histórias pregressas de vulnerabilidade para o desenvolvimento infantil até os dois anos de vida, tratamento adequado, suporte familiar, análises das fragilidades, assim como atividades que fomentam a inclusão e a qualidade de vida das pessoas com deficiências na localidade (BRASIL, 2012).

Geralmente, as UBS's possuem cobertura pré-definida a cada território uruguaianense, entretanto, o desempenho do serviço dispensado por elas às crianças com PC subsidia e sustenta evidências da escassez de ações e estratégicas da AB na RCPD local, porém, o Plano Municipal de Saúde (2018 - 2021) vem nos demonstrar algumas situações implícitas à Rede que ocorrem em outras políticas/programas de saúde na $A B$. Alguns desses se destacam na prevenção da deficiência no período da infância e juventude, sendo eles: o Serviço de Ginecologia - Obstétrica, o Programa Saúde e Prevenção na Escola, o Programa Bolsa Família e o Programa Primeira Infância Melhor (URUGUAIANA, 2017).

Em termos da AER da RCPD, todos os pais/responsáveis apontaram que seus dependentes com PC já utilizaram, no mínimo, um dos serviços disponíveis da Atenção Secundária na média complexidade do âmbito do SUS. Em Uruguaiana, esses serviços estão relacionados à RCPD por meio do CEO, da Policlínica Municipal, 
da Policlínica Infantil, do Setor de Fisioterapia, do Ambulatório de Especialidades do HSCCU, do Ambulatório de Saúde Mental e do TFD (URUGUAIANA, 2017).

No cenário da RCPD, nenhum dos pais/responsáveis relatou ter utilizado o CEO e o Ambulatório de Saúde Mental para tratamento de saúde de seus dependentes. Seria, aqui, mais um caso da falta de comunicação em saúde entre os setores da AER da RCPD de Uruguaiana? Provavelmente sim, visto que crianças com PC apresentam ocorrência de cárie, doença periodontal, má oclusão oral, alterações da cronologia de erupção dentária, bruxismo, lesões dentais traumáticas e másformações dentais, como a hipoplasia de esmalte (CESAR, 2007), e assim, acreditase que este público com PC necessitam de um local apropriado como o CEO para receberem assistência odontológica e cuidados preventivos para com a saúde bucal. Quanto à assistência na área de saúde mental, uma parte expressiva de pessoas com PC (75\%) apresentam déficit intelectual (BALADI; CASTRO; MORAIS FILHO, 2007) e, ao mesmo tempo, é necessário ter o psicólogo para assistir a criança e sua família frente às angustias, fantasias e medos, buscando a conscientização da realidade atual, trabalhando as limitações e valorizando as potencialidades do paciente em cada ciclo de vida (MADALENO et al., 2007).

Diante da não utilização do CEO e do Ambulatório de Saúde Mental, visualiza-se mais exemplos da fragmentação da RCPD municipal. Por outro lado, ações de outros serviços relacionados com a AER foram visualizadas nas descrições a seguir:

“[...] O tratamento com psicólogo, neurológico, fisioterapêutico, né! Na APAE e na Policlínica Municipal. [...] Consultei muitas vezes com neuro lá (Policlínica Municipal) [...]"<Responsável por Iris>.

"[...] Isso, no próprio hospital (tratamento de fisioterapia após a cirurgia do tendão calcâneo) [...]"< <esponsável por Hefesto $>$.

"[...] Ele fez fisio e fono (Policlínica Municipal) [...]" <Responsável por Hipnos>.

“[...] Foi na questão das órteses para ela ir a Bagé. [...]” <Responsável por Electra>.

"[...] Ir pra Porto Alegre pra consultar com o neuropediatra lá, porque não tem na cidade. [...] Foi feito lá uma ressonância com contraste [...]" < Responsável por Iris $>$. "[...] Sempre peguei cadeira de rodas para o Édipo através da Secretaria de Saúde [...]"<Responsável por Édipo>.

No geral foi percebido que a seção do TFD foi o serviço mais acionado pelos 16 pais/responsáveis das crianças com PC na AER, visto que todas elas receberam, em algum momento, uma órtese e/ou meios auxiliares de locomoção (cadeira de rodas e andadores) do serviço de referência da $10^{\mathrm{a}}$ Coordenadoria Regional de Saúde (CRS), sendo que o Centro Especializado em Reabilitação (CER) correspondente fica em Santa Maria, na $4^{\mathrm{a}}$ CRS. No passado, a referência para aquisição desses materiais era em Bagé, na $7^{\text {a }}$ CRS.

Na ação da AER, o Plano "Viver sem Limite" dispõe da premissa de aumentar o número de CER's para o favorecimento tanto no acesso quanto na qualidade dos procedimentos de saúde voltados às pessoas com deficiência no Brasil, principalmente na concessão de órteses, próteses e meios de locomoção (BRASIL, 2012). Recentemente, o aumento da concessão dessas tecnologias assistivas (TA) no RS, no pós-período do Plano (2012 - 2017), foi comprovado por Santos et al. 
(2017a).

Entretanto, o fator distância ainda é um entrave na vida das crianças com deficiência física e das suas famílias de Uruguaiana quanto ao acesso no CER de Santa Maria. Para a minimização desse entrave, um CER localizado em Alegrete, sede da $10^{a} \mathrm{CRS}$, está em fase de construção e possuirá o serviço especializado de reabilitação nas áreas física e auditiva. Futuramente, esse CER será referência para a população com deficiência dessas naturezas de Uruguaiana (RIO GRANDE DO SUL, 2017).

Imersos nesse entrave, alguns dos 16 pais/responsáveis das crianças com PC relataram almejar melhores condições de assistência à saúde para suas crianças em Uruguaiana e na $10^{\mathrm{a}} \mathrm{CRS}$, como foi visualizado nas descrições a seguir:

“[...] Um local que tivesse mais... um hospital, assim especializado. Com tudo lá dentro! Que tivesse fisioterapia, que tivesse a fonoaudiologia, consultas em geral, sabe? Eu acho que isso que falta aqui! Porque aqui é fronteira e poderia atender nossas crianças daqui, mas como também da Argentina, de repente de Libres (cidade de Passo de Los Libres) [...]. Não precisaria desse deslocamento tão grande, né! Daqui para Santa Maria, para Bagé, para São Borja ou para Porto Alegre para fazer uma coisa que poderia ter aqui! [...]" <Responsável por Ajax>.

"[...] Um centro maior que tivesse tudo que eles... o básico que eles precisam né! $A$ fisio, a fono, a psicopedagoga que não tem também, a fisio aquática [...]" <Responsável por Hipnos>.

A não oferta de um serviço infantil multiprofissional em Uruguaiana promove angústia. O conceito de oferecer à criança com PC um tratamento multidisciplinar é desejo desses pais/responsáveis, pois sabem da vantagem que essa forma de tratamento proporciona.

Essa vantagem foi relatada por Dias et al. (2010), em que 27 crianças com PC foram submetidas ao tratamento multidisciplinar de fisioterapia, fonoaudiologia, terapia ocupacional e pedagogia por quatro meses, sendo que esse conjunto de crianças teve uma evolução da função motora grossa e do desempenho motor. Para Borgneth (2004, p. 57), "o conceito de equipe multiprofissional atuando em modelo interdisciplinar é bastante interessante ao sugerir a possibilidade de interpenetração do conhecimento para suprir as necessidades do paciente".

De modo geral, a Portaria $n^{\circ} 793$ de 2012 do MS, que instituiu a RCPD no âmbito do SUS, traz essa ideia de multidisciplinaridade nos pontos de atenção a saúde (CER e CEO) que congregam os componentes da AER, a qual visa promover a equidade e ampliar o acesso aos usuários do SUS segundo as seguintes diretrizes: oferecer atenção integral, garantir conhecimento e orientação à pessoa com deficiência e ao seu núcleo familiar, fomentar a relação pessoa com deficiência e equipe de saúde e adequar os serviços oferecidos às necessidades dos usuários com deficiência (BRASIL, 2012).

Diante dessas diretrizes, o Plano Municipal de Saúde (2018 - 2021) demonstra que há lacunas nas ações de média complexidade relacionadas à RCPD em Uruguaiana. Percebe-se que o município não cumpre integralmente a saúde da criança com PC, erra no acesso de informação às famílias, não fomenta o vínculo pessoa com deficiência e equipe de saúde, visto não ter equipe multidisciplinar com 
ações interdisciplinares e, até o momento, o serviço de reabilitação municipal não está adequado em prol das crianças com PC.

Em relação à AHUE, sete pais/responsáveis mencionaram a utilização desse nível de atenção no suporte das crianças nascidas prematuras que permaneceram em Unidade de Terapia Intensiva (UTI) neonatal em Uruguaiana, além de internações por infecção em UTI infantil e por procedimentos cirúrgicos eletivos no $\mathrm{HSCCU}$, conforme pode ser percebido nas falas a seguir:

“[...] Isso, do lado esquerdo (cirurgia do tendão calcâneo)! Sim, em Uruguaiana! [...]. Tudo SUS [...]" <Responsável por Hefesto>.

"[...] Foram 28 dias de internação. Foi sei lá... acho que umas três semanas na UTI em Uruguaiana, né! [...]" <Responsável por Ajax>.

"[...] Foi na Policlínica, que ele tinha uma doutora [...] lá ela deu um papel e a gente baixamo (internação) para fazer a cirurgia no meio do saquinho, que tava querendo estrangular [...]"< Responsável por Aquiles $>$.

De modo geral, na RCPD, a AHUE tem a missão de receber, classificar o cuidado, expandir o acesso e qualificar a atenção à saúde das pessoas com deficiência nos setores hospitalares de urgência e emergência (BRASIL, 2012). Entretanto, o descontentamento com a RCPD quanto à execução de procedimentos cirúrgicos em ou fora de Uruguaiana foram narrados e podem ser visualizados nas descrições a seguir:

"[...] Em 2015 (cirurgia do alongamento do tendão calcâneo) [...]. No final de 2013 (solicitação da cirurgia em 2013) [...]" <Responsável por Hefesto>.

"[...] A demora para consultas é muito grande, a demora para cirurgias é muito grande, né! [...]” <Responsável por Ajax>.

Por fim, esses exemplos da $A B, A E R$ e AHUE da RCPD uruguaianense apontam oscilações de ações e estratégias na área da saúde, sendo que hora se tem articulação, hora se tem desarticulação, hora se vê sinergia, hora se vê assinergia que, de certo modo, esses exemplos negativos denigrem a imagem de uma Rede forte e eficaz pautada pela Portaria $n^{\circ} 793$ do MS, o que, de certa maneira, vem ao encontro do relato de Machado et al. (2018, p. 7):

É compreensível que sua proposta de integração entre as equipes que atuam nos diversos pontos de atenção, ainda não esteja de fato articulada, o que revela uma política ainda carente de atenção e investimentos, tanto da parte estrutural, no sentido de melhor dispor de tecnologias para prover $o$ atendimento adequado a essa população alvo, quanto na instrumentalização dos profissionais de cuidado em dispor de capacitações e conhecimentos acerca dessas incapacidades.

Assim, tudo isso culmina em uma evolução da RCPD sem sintonia nos níveis de $A B, A E R$ e AHUE, deformando a qualidade da atenção, a qualidade de vida infantil e familiar que imbrica em efeitos negativos à rede de cuidados à saúde geral, visto a subutilização dos recursos e da equidade em saúde (SANTOS; MARQUES; SOUZA, 2017). Dessa forma, a garantia da atenção integral do cidadão brasileiro 
com deficiência ainda está por ocorrer.

\subsection{Atores e/ou figuras ocultas da Rede de Cuidados da Pessoa com Deficiência de Uruguaiana:}

$\mathrm{Na}$ análise das entrevistas e estruturação dos resultados, foi percebido um expressivo número de citações relacionadas à Associação de Pais e Amigos dos Excepcionais (APAE) de Uruguaiana e ao Estágio Supervisionado de Fisioterapia em Neurologia Infantil (ESFNI) da Universidade Federal do Pampa (UNIPAMPA). Os 16 pais/responsáveis narraram esses dois setores que despendem ações de reabilitação, principalmente de fisioterapia para o público infantil com deficiência, como foi visualizado nas descrições a seguir:

"[...] Eu descobri que o Ajax tem paralisia cerebral com um ano e sete meses através da Eloá [...]" (Professora do Curso de Fisioterapia e Supervisora do Estágio em Fisioterapia em Neurologia Infantil da UNIPAMPA) <Responsável por Ajax>.

“[...] Édipo precisa de uma fisioterapia, algum acompanhamento e ele tem isso gratuito com a UNIPAMPA [...]"< <esponsável por Édipo>.

"[...]Eu fui abraçada juntamente com minha filha aqui na APAE, porque cheguei só com um encaminhamento neurológico e eu não sabia nem pra onde eu ia com aquele encaminhamento. [...] Fui avaliada pela fisioterapeuta, [...] neurologista, [...] psicólogo, [...] assistente social, [...] toda equipe! [...]" < Responsável por Iris>.

"[...] Daí lá (APAE) ele começou a fazer acompanhamento com neuro, a fisio, de lá já encaminharam para a fono, para o psicólogo. [...]" < Responsável por Hefesto $>$.

"[...] Foi através da UNIPAMPA que nós fomos para Bagé (CER) com ela! [...]" $<$ Responsável por Electra $>$.

Segundo o Estatuto da APAE de Uruguaiana (2012), um dos seus objetivos é prestar serviço de habilitação e reabilitação às pessoas com deficiência, preferencialmente as do tipo intelectual e múltipla, e com transtornos globais do desenvolvimento em qualquer ciclo de vida humano, na perspectiva do exercício da cidadania. Já o Projeto Pedagógico do Curso de Fisioterapia da UNIPAMPA aponta que o ESFNI promove atividades teóricas e práticas de assistência fisioterapêutica em habilitação e reabilitação ao público infantil com desordem do movimento humano, relacionadas às afecções do sistema nervoso central e periférico (UNIPAMPA, 2015a).

Dessa forma, as ações realizadas por essas duas instituições buscam a melhora do estado de saúde e da qualidade de vida desse público infantil e contribui com o processo de inclusão social a nível local. Entretanto, sabe-se que o serviço fisioterapêutico é uma parte de um todo no universo de reabilitação infantil.

A respeito do compromisso municipal em salvaguardar a saúde e a qualidade de vida das 16 crianças com PC, percebe-se que há transferência de responsabilidade, talvez passiva, do serviço de reabilitação infantil municipal para essas duas instituições, uma vez que a RCPD local não realiza seu papel na integridade do sujeito com deficiência. Acredita-se que essa ideia se naturalizou no município ao ponto de as famílias já buscarem diretamente, in loco, o suporte para tratamento de habilitação ou reabilitação das suas crianças, nesses locais como na 
descrição a seguir:

"[...] E aí, na hora eu pensei que onde tem criança especial é na APAE e se no caso da minha filha é neurológico então... ela é especial! E eu vim para cá e, prontamente, ela já recebeu avaliações. [...]" <Responsável por Iris>.

Sabe-se que os serviços de habilitação ou reabilitação na área da fisioterapia neurofuncional infantil desses dois setores não apresentam convênio com o SUS e, assim, toda gama de atendimentos é realizado sem a contrapartida financeira do MS. Entretanto, os encaminhamentos e as solicitações de procedimentos de saúde das crianças com deficiência (prescrição de exames e órteses e meios de locomoção) para a rede de atenção à saúde do município são costumeiramente executados.

Especificamente nas atividades do ESFNI, esse fato pode ser justificado por haver o Contrato Organizativo de Ação Pública Ensino - Saúde (UNIPAMPA, 2015b) celebrado entre a UNIPAMPA, a Prefeitura Municipal de Uruguaiana e a Secretaria Estadual de Saúde do RS na data de 11 de setembro de 2015. Esse viabiliza e garante a estrutura de serviços de saúde por cinco anos em condições de oferecer campo de prática mediante a integração ensino-serviço nas Redes de Atenção à Saúde.

Portanto, infere-se que esses dois serviços de reabilitação infantil, a APAE e o ESFNI, inter-relacionam-se com a RCPD, porém, não fazem parte oficialmente dela, ou seja, são configurados como atores e/ou figuras ocultas na RCPD de Uruguaiana. Afirma-se ainda que os procedimentos executados nesses dois locais não são contabilizados junto aos procedimentos de saúde do município, não descrevendo a realidade local quanto à presença da deficiência, o que faz gerar uma demanda não verdadeira e irreal quanto às necessidades do município na suplementação de procedimentos de saúde para essa parcela da população de Uruguaiana.

Dessa forma, com a leitura do Plano Municipal de Saúde $(2018$ - 2011) de Uruguaiana e as narrativas dos 16 pais/responsáveis de crianças com PC, foi possível construir uma hipotética RCPD no município com fluxos de ações e estratégias da $A B, A E R$ e AHUE, conforme apresentado na Figura 1:

Figura 1 - Hipotética Rede de Cuidados da Pessoa com Deficiência e seus fluxos de ações e estratégias na Rede de Atenção à Saúde de Uruguaiana 


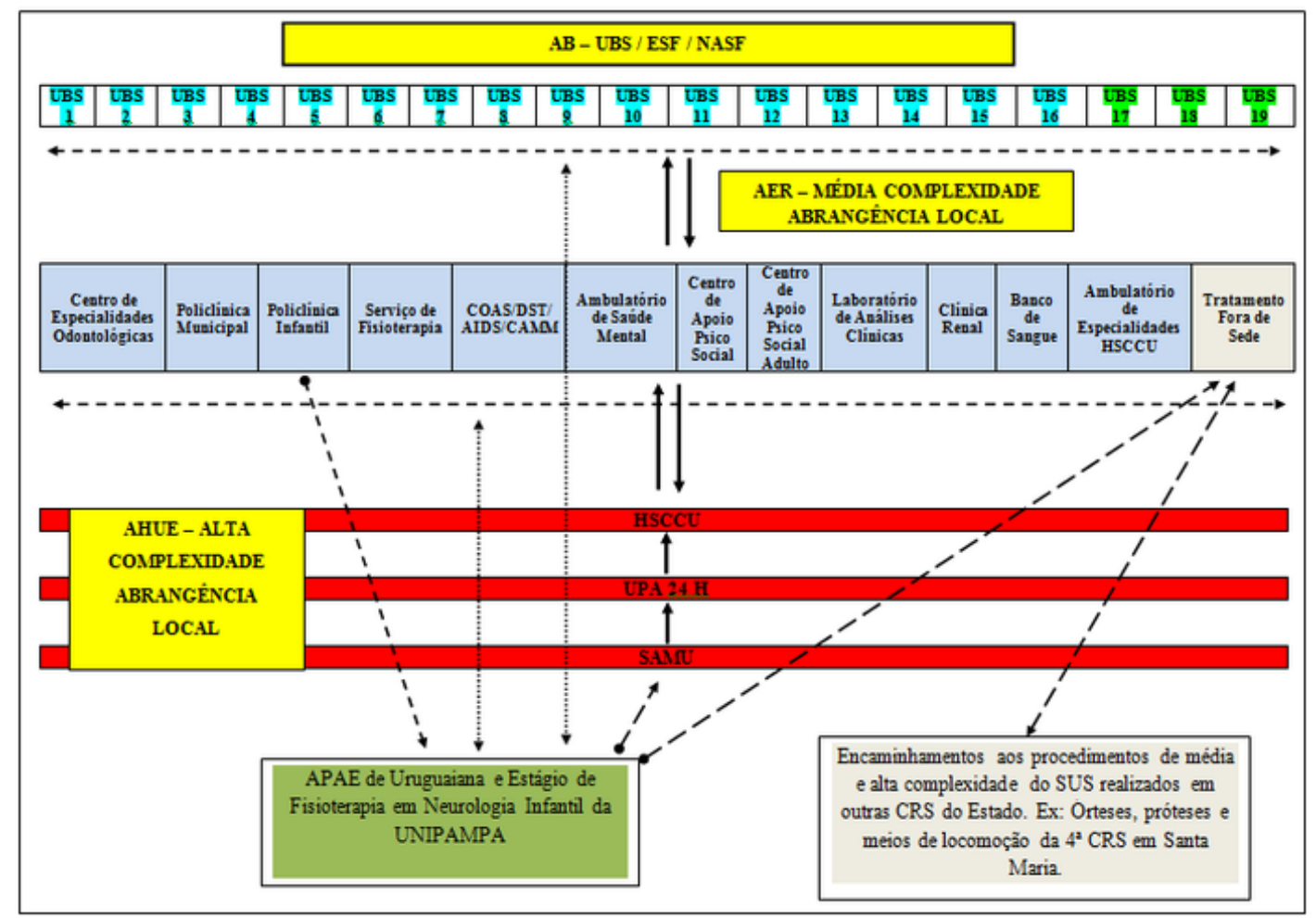

Legenda: AB: Atenção Básica; UBS: Unidade Básica de Saúde; ESF: Estratégia Saúde da Família; NASF: Núcleo de Apoio a Saúde da Família; AER: Atenção Especializada em Reabilitação; AHUE: Atenção Hospitalar de Urgência e Emergência; HSCCU: Hospital Santa Casa de Caridade de Uruguaiana; UPA: Unidade de Pronto Atendimento; SAMU: Serviço de Atendimento Móvel de Urgência; SUS: Sistema Único de Saúde; CRS: Coordenadoria Regional de Saúde; APAE: Associação de Pais e Amigos dos Excepcionais; UNIPAMPA: Universidade Federal do Pampa; COAS: Centro de Orientação e Aconselhamento Sorológico; CAMMI: Centro de Aplicação e Monitoramento de Medicamentos Injetáveis; DST: Doença Sexualmente Transmissível e AIDS: Síndrome da Imunodeficiência Humana. Fonte: conforme dados do autor (2019), baseado nos relatos dos 16 pais/responsáveis de crianças com PC e no Plano Municipal de Saúde de Uruguaiana (2018 - 2021)

A articulação de ações em saúde realizada pela APAE de Uruguaiana e pelo ESFNI da UNIPAMPA pode ocorrer em todos os níveis de atenção de saúde do município. Tais setores são componentes transformadores da realidade local, quanto ao processo de melhorias no modelo de assistência à saúde dos munícipes com deficiência. Entende-se que tais ações surgiram das necessidades desta população específica e pelos objetivos institucionais similares que estes dois setores de reabilitação buscam: melhorar a qualidade de vida da população com deficiência da região com vistas a integralidade do cuidado, como sugerido pela RCPD, além de facilitar o processo de inclusão social.

\subsection{Interferência da Rede de Cuidados da Pessoa com Deficiência na inclusão social de crianças com paralisia cerebral em Uruguaiana:}

Nesses seis últimos anos, a RCPD nacional vem se edificando, expandindo e desenvolvendo o acesso da pessoa com deficiência aos serviços de saúde no âmbito do SUS, em especial nos serviços de habilitação e reabilitação. Em 2017, ela já possuía 196 CER's, 35 Oficinas Ortopédicas (OO) e 551 CEO's (477 ativos e 74 suspensos) (BRASIL, 2018). 
No RS, até outubro de 2017, a RCPD possuía seis CER's em funcionamento e outros cinco em fase de conclusão, e três OO's (RIO GRANDE DO SUL, 2017). O objetivo da Rede estadual é "promover a saúde e reabilitar as pessoas com deficiência por meio de suas capacidades funcionais (física, auditiva, intelectual e visual)" (RIO GRANDE DO SUL, 2016, p. 122).

Nesse panorama, a RCPD trouxe benefícios aos gaúchos com deficiências, em que o total da oferta de primeira consulta na área de reabilitação em 2015 ultrapassou o número de 21.800 consultas (RIO GRANDE DO SUL, 2016). Quanto à prescrição e entrega de órteses, próteses e meios de locomoção, de 2008 a 2016, o estado apresentou evolução substancial e estatisticamente significativa ano após ano ( $r=0,99 ; p<0,0001)$ para a população com deficiência e chegou a mais de 134 mil entregues nesse período (SANTOS et al., 2017a).

Em Uruguaiana, todos os pais/responsáveis sinalizaram o uso dos benefícios do CER da RCPD pelas crianças com PC, com destaque para o recebimento de órteses e de meios de locomoção, sendo que 10 crianças receberam cadeira de rodas infantil. Segundo Costa et al. (2010), a cadeira de rodas contribui para a mobilidade do corpo, a fluidez dos movimentos e o deslocamento. É um dos equipamentos que contribui para a independência funcional e autonomia do ser humano com deficiência. Globalmente, tanto as órteses como a cadeira de rodas são conhecidas como TA. Essa se caracteriza por ser:

\footnotetext{
Uma área do conhecimento, de característica interdisciplinar, que engloba produtos, recursos, metodologias, estratégias, práticas e serviços que objetivam promover a funcionalidade, relacionada à atividade e participação, de pessoas com deficiência, incapacidades ou mobilidade reduzida, visando sua autonomia, independência, qualidade de vida e inclusão social (CAT, 2007, p. 3).
}

Refletindo sobre as TA's, a crescente concessão desses insumos no RS ancora-se no aumento da quantidade de CER's no estado junto à AER da RCPD e à presença indispensável de uma equipe multidisciplinar com ações interdisciplinares de trabalho em habilitação e reabilitação. Por outro, Galvão, Barroso e Grutt (2013) enfatizam que o chancelamento e a regulamentação dos profissionais da Terapia Ocupacional e da Fisioterapia para prescreverem esses materiais relacionados ao ato não cirúrgico no âmbito do SUS, potencializou favoravelmente essa ação.

Ao constatarem a necessidade de prescrever a TA para a criança com deficiência, é rotina da equipe da APAE de Uruguaiana e do ESFNI fazer o encaminhamento para o setor de TFD da Secretaria Municipal de Saúde de Uruguaiana, o qual protocola a solicitação e o encaminha para o serviço de referência estadual. O benefício do uso desses dispositivos foi expresso pelos pais/responsáveis dessas crianças da seguinte forma:

"[...] Eu só vejo pontos positivos, porque ajuda no desenvolvimento e na locomoção dela. [...]. A órtese mantém a postura dos pés dela, então tudo ajuda! A cadeira também para a locomoção... ajuda, facilita bastante o movimento dela, a locomoção. [...]" < Responsável por Héstia>.

"[...] Foi encaminhado pra por uma órtese no pé... no pé esquerdo e um abdutor do polegar esquerdo, [...]. Agora ela passou pra palmilha [...]! Se ela, no caso, não faz 
uso da palmilha ela cai. [...]"< Responsável por Iris>.

A partir desse salvo-conduto familiar, as TA's são sublinhadas como recursos indispensáveis aos profissionais que lidam com reabilitação da criança com PC. Portanto, concorda-se com Varela e Oliver (2013) que descrevem que a ela, aliada ao seu bom uso e à utilização de forma sábia, proporciona múltiplas situações que estimulam a maturação, as relações sociais, a escolarização, a participação e a inclusão social, mas não garante a realização de atividades de vida diária e prática por si só.

Para tanto, a família tem que ter consciência dos benefícios e promover o seu uso rotineiramente junto à criança. Por sua vez, Santos et al. (2017b) afirmam que por estimular a autonomia e a independência, a TA se relaciona intimamente com a qualidade de vida das pessoas com deficiência.

A relação entre qualidade de vida da criança com PC e o uso da TA fica demonstrada ao mensurá-la por meio do Questionário de Qualidade de Vida para Crianças com PC (CP QOL Child), criado pelo CP QOL Group (2013). Nesse, existem duas dimensões: acesso a serviços (saúde, educação e lazer) e equipamento especial (órteses, próteses e meios auxiliares de locomoção), em que suas pontuações somadas a outras seis dimensões resultam em uma pontuação final que denota o nível de qualidade de vida da criança com PC.

Nessa vertente, é esperado que ao apresentar acessos satisfatórios aos serviços de saúde e às TA's, o nível de qualidade de vida da criança com PC seja influenciado positivamente por ambas as dimensões. Caso ocorra o contrário, Vasconcelos et al. (2014) afirmam que uma dimensão do CP QOL Child afeta diretamente a outra, depreciando o nível de qualidade de vida da criança com PC. Baseado nessas considerações, o desempenho dos serviços de saúde da RCPD pode afetar, positivamente ou negativamente, a qualidade de vida das crianças com PC.

Nas descrições das crianças com PC citadas anteriormente, os benefícios pelo uso da TA se relacionaram à facilidade na mobilidade urbana, minimização da sobrecarga de peso à família, recurso para a escolarização e no ajuste corpóreo infantil.

Outra sinalização do impacto favorável da RCPD em Uruguaiana foi relatada por um pai/responsável relacionada ao nível da AHEU local. Uma criança com PC apresentava pé equino causado pela alteração tônica, a hipertonia. Assim, por meio de um procedimento cirúrgico realizado no HSCCU, foi realizado o realinhamento articular do tornozelo esquerdo e a correção do apoio do pé dessa criança. A necessidade da cirurgia foi sustentada por um prognóstico funcional de deambulação favorável, mas contribuiu indiretamente para a reconstrução da imagem de um corpo anormal, conforme demostrado a seguir:

“[...] Melhorou bastante (aceitação após a cirurgia do alongamento de tendão calcâneo)! Porque antes mexiam com ele por causa do pé, agora já não! Agora ele nem dá bola! [...] Chamavam: olha o pezinho! Olha o pezinho torto! Principalmente na escola! [...]" < Responsável por Hefesto >.

A deficiência física é exteroceptiva, como referenda Foucault (2013, p. 7): "Meu corpo é o contrário da utopia". Esse significado demonstra que o corpo não é 
fantasia, mas é concreto e real, e no caso da criança com PC, carrega as marcas e sinais de ser heterogênea perante os demais. Em uma categorização, essa criança demonstra possuir um estigma, que para Goffman (1988), transita pela ideia do defeituoso e do fraco por ter uma desvantagem corpórea e com isso afeta sua aceitação social. Quanto à cirurgia do pé, houve uma correção e um realinhamento corporal que ofuscou sua marca social.

Ao se comparar o recurso cirúrgico com o recurso da TA, há uma contradição quanto à questão do estigma. O primeiro apresenta a ideia do modelo biomédico em que a criança pode ter "consertada" sua marca social, enquanto que o segundo, como Santos et al. (2017b) citam, o uso da TA não só revela, mas também exacerba o estigma publicamente, o que faz a diferença ser notada com maior facilidade. Tal fato foi visualizado nas descrições de pais/responsáveis das crianças com PC a seguir:

"[...] Muitas crianças me perguntam [...] ele tá doente? Eu digo não! Aí elas me perguntam: por que ele tá nessa cadeira? Aí eu digo que está na cadeira porque ele não consegue caminhar, mas não porque ele está doente! [...]" < Responsável por Ajax>.

"[...] Esperando o ônibus e um senhor veio e perguntou para mim se ele caminhava, sendo que ele estava na cadeira de rodas! [...] Aí eu disse pra ele assim: sim ele caminha, porém ele está na cadeirinha para chamar a atenção das pessoas! [...]" $<$ Responsável por Poseidon>.

Nesse sentido, espera-se que os dois recursos, a cirurgia e a TA, possam promover a superação de certos entraves na vida dessas crianças com PC, como por exemplo, os obstáculos físicos, a discriminação, a opressão e a cultura da homogenia que potencializam o modelo social da deficiência (DINIZ; BARBOSA; SANTOS, 2009).

Assim, contribuindo com o universo da atenção à saúde da pessoa com deficiência, a Rede de Cuidados tece novos nós de articulações em toda esfera social que vão desenhando um novo horizonte e, esses nós, logicamente, precisam ser vistoriados sempre, reavaliados e novamente apertados, mas nunca desatados. Certamente, o caminho da inclusão social é uma reta de infinitos pontos.

\section{Considerações finais}

O cenário uruguaianense apresenta falhas de atenção à saúde das crianças com PC em todos os níveis (inicia na $A B$, transpassa a AER e culmina na AHUE) da RCPD que pertence ao eixo saúde do Plano "Viver sem Limite" conforme relatos dos pais/responsáveis de crianças com PC.

Há uma incompleta assistência da rede de saúde para com as crianças com PC, fato percebido pela não citação, estruturação e detalhamento da RCPD no Plano Municipal de Saúde (2018 - 2021) em Uruguaiana.

Ao mesmo instante existe uma insuficiência de profissionais fundamentais (neuropediatras, fisioterapeutas, terapeutas ocupacionais, fonoaudiólogos, entre outros) que compõem uma equipe interdisciplinar para executar ações e estratégias 
relacionadas com a saúde da pessoa com deficiência, em especial, a criança com PC. Esses profissionais desfalcam a RCPD e impedem uma melhor interlocução entre suas estruturas, prejudicando os usuários que dela dependem.

Ficou evidenciada a importância da APAE de Uruguaiana e do ESFNI da UNIPAMPA no processo de habilitação e reabilitação infantil no cenário local, os quais configuraram como atores e/ou figuras ocultas da RCPD.

Mesmo com o não conhecimento da existência Plano "Viver sem Limite" e da RCPD pelos 16 pais/responsáveis de crianças com PC de Uruguaiana, vislumbrouse que houve uma positividade no processo inclusão social, onde a AER foi o ponto de articulação da Rede que mais se destacou com a prescrição de TA's a esse público infantil com deficiência.

Por fim, há um descompasso na assistência em saúde da RCPD de Uruguaiana, ou seja, existe uma não integralidade do cuidado em saúde, que interfere negativamente o processo de reabilitação e de inclusão social dessas 16 crianças com PC.

\section{REFERÊNCIAS}

ALVES, M. A.; RIBEIRO, F. F.; SAMPAIO, R. F. Potencial de mudança nas práticas de saúde: a percepção de trabalhadores de uma Rede de Reabilitação em (trans)formação. Fisioterapia e Pesquisa, São Paulo, v. 23, n. 2, p. 185-192, 2016.

APAE - ASSOCIAÇÃO DE PAIS E AMIGOS DOS EXCEPCIONAIS DE URUGUAIANA. Estatuto da APAE de Uruguaiana. Uruguaiana, 2012.

BALADI, A. B. P. C.; CASTRO, N. M. D.; MORAES FILHO, M. C. Paralisia Cerebral. In: FERNADES, A. C. et al. AACD - Medicina e Reabilitação Princípios e Prática. 1. ed. São Paulo: Artes Médicas, 2007. cap. 2.

BORGNETH, L. Considerações sobre o processo de reabilitação. Acta Fisiátrica. São Paulo, v. 11, n. 2, p. 55-59, 2004.

BRASIL. Ministério da Mulher, da Família e dos Direitos Humanos. Ministra participa, em Uruguaiana (RS), de seminário sobre tráfico de seres humanos. Brasília, 2013a. Disponível em: <https://www.mdh.gov.br/sdh/noticias/2013/agosto/ministra-participa-emuruguaiana-rs-de-seminario-sobre-trafico-de-seres-humanos>. Acesso em: 03 mai. 2019.

. Ministério da Saúde. Portaria $\mathrm{n}^{\circ}$ 2488, de 21 de outubro de 2011. Brasília, 22 de outubro de 2011b. Disponível em: <http://bvsms.saude.gov.br/bvs/saudelegis/gm/2011/prt2488_21_10_2011.html>. Acesso em: 03 mai. 2019.

. Ministério da Saúde. Portaria $n^{\circ}$ 793, de 24 de abril de 2012. Brasília, 25 de abril de 2012a. Disponível em: 
<http://bvsms.saude.gov.br/bvs/saudelegis/gm/2012/prt0793_24_04_2012.html>. Acesso em: 03 mai. 2019.

. Ministério da Saúde. Relatório de Gestão - Exercício 2017. Brasília: Ministério da Saúde, Secretaria de Atenção à Saúde, 2018. Disponível em: <https://www.saude.gov.br/relatorio-de-gestao>. Acesso em: 03 mai. 2019.

. Ministério da Saúde. Resolução n 466, de 12 de dezembro de 2012. Brasília, 12 de dezembro de 2012b. Disponível em: < https://bvsms.saude.gov.br/bvs/saudelegis/cns/2013/res0466_12_12_2012.html>. Acesso em 03 mai. 2019.

. Ministério da Saúde. Secretaria de Atenção à Saúde. Departamento de Ações Programáticas Estratégicas. Diretrizes de atenção à pessoa com paralisia cerebral / Ministério da Saúde, Secretaria de Atenção à Saúde, Departamento de Ações Programáticas Estratégicas. - Brasília: Ministério da Saúde, 2013b. Disponível em:

$<$ https://bvsms.saude.gov.br/bvs/publicacoes/diretrizes_atencao_paralisia_cerebral. pdf>. Acesso em: 03 mai. 2019.

- Ministério da Saúde. Secretaria de Atenção à Saúde. Departamento de Atenção Básica. Nota Técnica. Assunto: Informações sobre as ações e programas do Departamento de Atenção Básica. Município: Uruguaiana, RS. Disponível em: <http://dab2.saude.gov.br/sistemas/notatecnica/frmListaMunic.php>. Acesso em: 01 abr. 2019.

. Presidência da República. Casa Civil. Subchefia para Assuntos Jurídicos. Decreto que institui o Plano Nacional dos Direitos da Pessoa com Deficiência - Plano Viver Sem Limite. n. 7612, de 17 de novembro de 2011. Brasília, 18 de novembro de 2011a. Disponível em: <http://www.planalto.gov.br/ccivil_03/_ato20112014/2011/decreto/d7612.htm>. Acesso em: 03 mai. 2019.

CAT - COMITÊ DE AJUDA TÉCNICA. Ata VII Reunião do Comitê de Ajudas Técnicas CAT CORDE/SEDH/PR. Brasília, 2007. Disponível em: <http://www.infoesp.net/CAT_Reuniao_VII.pdf>. Acesso em: 03 mai. 2019.

COIMBRA, C. da S.; MÜLLER, A. B. Comprometimento funcional, condição socioeconômica e sobrecarga de cuidado na paralisia cerebral. R. Bras. Qual. Vida, Ponta Grossa, v. 9, n. 3, p. 208-222, jul./set. 2017.

COSTA, V. de S. P. et al. Representações sociais da cadeira de rodas para a pessoa com lesão da medula espinhal. Revista Latino-Americana de Enfermagem, Ribeirão Preto, v. 18, n. 4, ago. 2010.

CP QOL GROUP. CP QOL Cerebral Palsy Quality of Life - Manual Version: $2^{\text {a Version. }}$ Cerebral Palsy Quality of Life. Melbourne, Austrália, 2013. Disponível em: <http://www.cpqol.org.au>. Acesso em: 29 abr. 2019. 
DANTAS, M. S. de A. et al. Atenção profissional à criança com paralisia cerebral e sua família. Revista Enfermagem UERJ, Rio de Janeiro, v. 25, n. e18331, p. 1-6, ago. 2017.

DIAS, A. C. B. D et al. Desempenho funcional de crianças com paralisia cerebral participantes de tratamento multidisciplinar. Fisioterapia e Pesquisa, São Paulo, v. 17, n. 3, p. 225-229, jul./set. 2010.

DINIZ, D.; BARBOSA, L.; SANTOS, W. R. Deficiência, direitos humanos e justiça. Sur, Revista Internacional de Direitos Humanos, São Paulo, v. 6, n. 11, p. 64-77, dez. 2009.

DUBOW, C.; GARCIA, E. L.; KRUG, S. B. F. Percepções sobre a Rede de Cuidados à Pessoa com Deficiência em uma Região de Saúde. Saúde em Debate, Rio de Janeiro, v. 42, n. 117, p. 455-467, abr./jun. 2018.

FIGUEIREDO, N. M. A. Entre a filosofia e as políticas públicas: o que saber sobre o SUS. In: FIGUEIREDO, N. M. A.; TONINI, T. (Org.). SUS e PSF para enfermagem: práticas para o cuidado em saúde coletiva. São Caetano do Sul: Yendis, 2007.

FOUCAULT, M. O corpo Utópico, As heterotopías: Michel Foucault; Posfácio de Daniel Defert. Tradução Salma Tannus Muchail. São Paulo: n-I Edições, 2013.

GALVÃO, C. R. C.; BARROSO, B. I. de L.; GRUTT, D. de C. A tecnologia assistiva e os cuidados específicos na concessão de cadeiras de rodas no Estado do Rio Grande do Norte. Cadernos Brasileiros de Terapia Ocupacional UFSCar, São Carlos, v. 21, n. 1, p. 11-18, 2013.

GOFFMAN, E. Estigma: notas sobre a manipulação da identidade deteriorada. 4. ed. Rio de Janeiro: LTC Editora, 1988.

MACHADO, W. C. A. et al. Integralidade na rede de cuidados da pessoa com deficiência. Texto contexto - enferm., Florianópolis, v. 27, n. 3, e4480016, 2018.

MADALENO, I. M. P. et al. Psicologia na reabilitação infantil e adultos. In: FERNADES, A. C. et al. AACD - Medicina e Reabilitação Princípios e Prática. 1. ed. São Paulo: Artes Médicas, 2007, cap. 42.

MENDES, V. L. F. Saúde Sem Limite: implantação da Rede de Cuidados à Saúde da Pessoa com Deficiência. Divulgação em Saúde para Debate, Rio de Janeiro, v. 52, p. 146-152, out. 2014.

MINAYO, M. C. de S. A. O desafio do conhecimento: pesquisa qualitativa em saúde. 14. ed. São Paulo: Hucitec, 2014.

OTHERO, M.B.; AYRES, J.R.C.M. Healthcare needs of people with disabilities: subjects' perspectives through their life histories. Interface - Comunic., Saude, Educ., v.16, n.40, p.219-33, jan./mar. 2012. 
OTHERO, M. B.; DALMASO, A. S. W. Pessoas com deficiência na atenção primária: discurso e prática de profissionais em um centro de saúde-escola. Interface (Botucatu), Botucatu, v. 13, n. 28, p. 177-188, jan./mar. 2009.

URUGUAIANA. Prefeitura Municipal. Secretaria Municipal de Saúde. Plano Municipal de Saúde 2018 - 2021. 2017.

PRODANOV, C. C.; FREITAS, E. C. de. Metodologia do trabalho científico: métodos e técnicas de pesquisa e do trabalho acadêmico. 2. ed. Novo Hamburgo: Feevale, 2013.

RIO GRANDE DO SUL. Secretaria de Saúde. Anexo Resolução n 454/17 - CIB - RS. Plano Estadual - Rede de Cuidados à Pessoa com Deficiência do Rio Grande do Sul. Porto Alegre, 2017. Disponível em: <https://saude.rs.gov.br/saude-da-pessoa-comdeficiencia>. Acesso em: 29 abr. 2019.

Secretaria da Saúde. Plano Estadual de Saúde 2016 - 2019. Porto Alegre, 2016. Disponível em: <https://saude.rs.gov.br/plano-estadual-de-saude>. Acesso em: 04 mai. 2019.

. Secretaria de Saúde. Saúde da Pessoa com Deficiência: Redes, serviços endereços e mapas. Porto Alegre, 2019. Disponível em: <https://saude.rs.gov.br/saude-da-pessoa-com-deficiencia>. Acesso em: 04 mai. 2019 .

ROSENBAUM, P. et al. A report: the definition and classification of cerebral palsy. April 2006. Dev Med Child Neurol, v. 49, n. 109, p. 8-14, fev. 2007.

ROTTA, N. T. Paralisia cerebral, novas perspectivas terapêuticas. Jornal de Pediatria (Rio J.), Porto Alegre, v. 78, n. supl. 1, p. S48-S54, ago. 2002.

SANTOS, C. C. et al. Política pública, deficiência física, concessão de órteses, próteses e meios de locomoção no Rio Grande do Sul: período pré/pós Plano Viver Sem Limite. Interfaces Científicas, Aracaju, v. 5, n. 3, p. 17-26, $2017 a$.

SANTOS, R. et al. Tecnologia assistiva e suas relações com a qualidade de vida de pessoas com deficiência. Revista de Terapia Ocupacional da Universidade de São Paulo, v. 28, n. 1, p. 54-62, 8 jun. 2017b.

SANTOS, K. H. dos; MARQUES, D.; SOUZA, A. C. de. Crianças e adolescentes com paralisia cerebral: análise sobre longitudinalidade do cuidado. Texto \& Contexto Enfermagem, Florianópolis, v. 26, n. 2, 2017. e00530016.

SASSAKI, R. K. Inclusão: acessibilidade no lazer, trabalho e educação. Revista Nacional de Reabilitação (Reação), São Paulo, p. 10-16, mar./abr. 2009. Ano XII.

SCHULTZ, T. G; ALONSO, C. M. do C. Cuidado da criança com deficiência na Atenção Primária à Saúde. Cad. Ter. Ocup. UFSCar, São Carlos, v. 24, n. 3, p. 611-619, 2016. 
TEIXEIRA, J. A. C. Comunicação em saúde: Relação Técnicos de Saúde - Utentes. Aná. Psicológica, Lisboa, v. 22, n. 3, p. 615-620, set. 2004.

UNIPAMPA - UNIVERSIDADE FEDERAL DO PAMPA. Projeto Pedagógico do Curso de Fisioterapia 2015. Uruguaiana, 2015a.

UNIPAMPA - UNIVERSIDADE FEDERAL DO PAMPA. Termo de Contrato Organizativo de Ação Pública Ensino - Saúde. Uruguaiana, 11 de setembro de 2015. 2015b.

VASCONCELOS, V. M. et al. Domínios de qualidade de vida de crianças com paralisia cerebral: estudo transversal. Online Brazilian Journal of Nursing, Niterói, v. 13, n. 4, p. 613621, dez. 2014.

VARELA, R. C. B.; OLIVER, F. C. A utilização de Tecnologia Assistiva na vida cotidiana de crianças com deficiência. Ciênc. saúde coletiva, Rio de Janeiro, v. 18, n. 6, p. 1773-1784, jun. 2013.

Christian Caldeira Santos. Doutorado em Diversidade Cultural e Inclusão Social (FEEVALE). Universidade Federal do Pampa (UNIPAMPA). Professor Adjunto nível II do Curso de Fisioterapia. BR 472 - Km 585, Caixa Postal 118, Uruguaiana, Rio Grande do Sul, Brasil. <christiansantos@unipampa.edu.br>.

Jacinta Sidegum Renner. Doutorado em Engenharia de Produção (UFRGS), com ênfase em Ergonomia. Universidade Feevale. Professora do Programa de PósGraduação em Diversidade Cultural e Inclusão Social. Campus II, ERS-239, 2755, Novo Hamburgo, Rio Grande do Sul, Brasil.<jacinta@feevale.br>.

Gustavo Roese Sanfelice. Doutorado em Ciências da Comunicação (UNISINOS). Professor do Programa de Pós-Graduação em Diversidade Cultural e Inclusão Social. Campus II, ERS-239, 2755, Novo Hamburgo, Rio Grande do Sul, Brasil. <sanfeliceg@feevale.br>.

Susane Graup. Doutorado em Engenharia de Produção (UFSC), com ênfase em Ergonomia. Universidade Federal do Pampa (UNIPAMPA). Professora Adjunta nível IV do Curso de Educação Física. BR 472 - Km 585, Caixa Postal 118, Uruguaiana, Rio Grande do Sul, Brasil. <susanegraup@unipampa.edu.br>.

Rodrigo de Souza Balk. Doutorado em Ciências Biológicas (UFSM), com ênfase em Bioquímica Toxicológica. Universidade Federal do Pampa (UNIPAMPA). Professor Associado nível II do Curso de Fisioterapia. BR 472 - Km 585, Caixa Postal 118, Uruguaiana, Rio Grande do Sul, Brasil. <rodrigobalk@unipampa.edu.br>. 
Como citar: SANTOS, Christian Caldeira et al. Plano "Viver sem Limite" e a Rede de Cuidados da Pessoa com Deficiência: representações em um cenário da Fronteira Oeste do Rio Grande do Sul. Redes (St. Cruz Sul, Online), Santa Cruz do Sul, v. 25, p. 2760-2782, dez. 2020. ISSN 1982-6745. doi:https://doi.org/10.17058/redes.v25i0.14905.

\section{CONTRIBUIÇÃO DE CADA AUTOR}

Conceituação (Conceptualization): Christian Caldeira Santos e Jacinta Sidegum Renner. Análise Formal (Formal analysis): Christian Caldeira Santos e Jacinta Sidegum renner. Obtenção de Financiamento (Funding acquisition): Christian Caldeira Santos Investigação/Pesquisa (Investigation): Christian Caldeira Santos Metodologia (Methodology): Christian Caldeira Santos, Jacinta Sidegum Renner, Gustavo Roese Sanfelice, Susane Graup e Rodrigo de Souza Balk.

Supervisão/orientação (Supervision): Jacinta Sidegum Renner.

Escrita - Primeira Redação (Writing - original draft): Christian Caldeira Santos e Jacinta Sidegum Renner.

Escrita - Revisão e Edição (Writing - review \& editing): Christian Caldeira Santos, Jacinta Sidegum Renner, Gustavo Roese Sanfelice, Susane Graup e Rodrigo de Souza Balk.

Fontes de financiamento: apoio da Coordenação de Aperfeiçoamento de Pessoal de Nível Superior - Brasil (CAPES), pelo Programa Suporte à Pós-Graduação de Instituições de Ensino Particulares (PROSUC), Modalidade II. 